\title{
THE MERGER OF A HELIUM STAR AND A BLACK HOLE: GAMMA-RAY BURSTS
}

\author{
Weiqun Zhang and Chris L. Fryer \\ UCO/Lick Observatory, University of California \\ Santa Cruz, CA 95064 \\ zhang@ucolick.org and cfryer@ucolick.org
}

\begin{abstract}
There is growing observational evidence that gamma-ray bursts (GRBs) are powered by black holes accreting rapidly through a disk. The supernova-like outburst that accompanies some gamma-ray bursts suggest that some long-duration GRBs may be driven by the accretion of a rotating stellar core onto a central black hole. Such a system can be produced when a compact remnant spirals into the core of its binary companion. During the inspiral, orbital angular momentum is injected into the helium core. By the time the compact remnant reaches the center of the helium core, it too has gained angular momentum as well as mass, producing a rapidly accreting black hole (or neutron star) at the center of a rotating stellar core.

Whether or not such a merger (termed He-merger) can produce a GRB depends upon the initial mass and spin of the central black hole, as well as the angular momentum in the stellar core. In this paper, we use a 3-dimensional smooth particle hydrodynamics (SPH) code to follow the He-merger process and make quantitative estimates of the initial mass and spin of the central compact remnant, as well as the angular momentum in the accreting helium core. During the inspiral, a $2 M_{\odot}$ compact remnant gains $\sim 0.5-3.5 M_{\odot}$ depending on the mass of the helium core (more massive cores provide more accretion). The accretion rates on the central remnant are initially very high, $10^{5} M_{\odot} \mathrm{yr}^{-1}$ up to $10^{6} M_{\odot} \mathrm{yr}^{-1}$ (the accretion rate increases with increasing helium star mass), and the central remnant quickly becomes a black hole if it was not one already.

From these accretion rates, we estimate GRB explosion energies. In all mergers, magnetically driven jets are expected to produce GRB explosions with energies above $10^{51}$ ergs. For neutrino-annihilation-driven explosions, the GRB energy increases dramatically with helium star mass: the merger of a $2 M_{\odot}$ compact remnant with a $4 M_{\odot}$ helium star only produces a $10^{47} \mathrm{erg}$ explosion in $\sim 500 \mathrm{~s}$ whereas the merger of a $2 M_{\odot}$ compact remnant with a $16 M_{\odot}$ helium star produces a $>10^{52} \mathrm{erg}$ explosion in $\sim 65 \mathrm{~s}$.
\end{abstract}

Subject headings: gamma-rays: bursts - binaries: close - black holes 


\section{Introduction}

Although there is no agreed upon engine powering gamma-ray bursts(GRBs), models based upon black hole accretion disks (BHAD models) are attractive. The progenitors of this class of GRB engine include the mergers of compact binaries (double neutron star, black hole and a neutron star, white dwarf and a black hole), collapsars, and the inspiral of a neutron star or black hole into a helium star (see Fryer, Woosley, \& Hartmann 1999a for a review). In all of these cases, these progenitors produce accretion disks around black holes which, as they accrete, use neutrinos or magnetic fields to convert part of the gravitational potential or rotational energy of the disk material (or black hole) into a GRB outburst. The BHAD systems formed by these progenitors differ mainly in the disk mass, disk angular momentum, or amount of material above the plane of the rotation disk and these variations may well explain the diversity of the observed sample of gamma-ray bursts.

For instance, classical GRBs seem to divide into long and short duration subgroups (e.g. Kouveliotou et al. 1993; Katz \& Canel 1996; Tavani 1998), and it is thought that these two distinct subgroups are explained by different BHAD progenitors (e.g. Fryer et al. 1999a). Three progenitors may produce long-duration GRBs: mergers of white dwarfs with black holes, collapsars, and He-mergers. The disks formed in the mergers of white dwarfs with black holes (or neutron stars $^{1}$ ) represent one extreme in disk characteristics. Of the three long-duration BHAD GRB progenitors, white dwarf mergers produce disks with the most angular momentum and the least amount of material along the rotation axis. Although the high angular momentum in these disks make neutrino-mediated explosions extremely weak, white dwarf/black hole mergers may produce strong GRB explosions via some magnetic field mechanism (Fryer et al. 1999b). Collapsars, on the other hand, represent the other extreme: they have less angular momentum and much more material along the axis of rotation (MacFadyen \& Woosley 1999; MacFadyen, Woosley ,\& Heger 2000). Since the connection between GRB 980425 and supernova 1998bw (Kulkarni et al. 1998), which is naturally explained by collapsar models (Woosley 1993; MacFadyen \& Woosley 1999; MacFadyen et al. 2000), the collapsar model has become the favorite progenitor for long-duration bursts.

But can all long-duration gamma-ray bursts be collapsars? There is increasing evidence to suggest that collapsars cannot explain all GRBs. First, the X-ray and optical lightcurves of GRBs suggest that the environment around the GRB engine is sometimes best fit by a wind-swept model, and other times is best fit by a constant density model (Chevalier \& Li 2000, Livio \& Waxman 2000). Based on the fact that collapsars occur only in massive stars, Chevalier \& Li (2000) and Livio \& Waxman (2000) both argue that those GRBs best fit by constant density environments

\footnotetext{
${ }^{1}$ Belczyński, Bulik, \& Rudak (2000) have found that white dwarf/neutron star mergers, which may be able to produce GRB outbursts using a magnetic-field driven mechanism, dominate the number of white dwarf mergers.
} 
must not be collapsars ${ }^{2}$. In addition, collapsars may have trouble explaining the iron emission lines detected in three GRBs: GRB 970508 (Piro et al. 1999), GRB 970828 (Yoshida et al. 1999), and GRB 991216 (Piro, et al. 2000). To explain these detections (the detections in GRB 970508 and GRB 970828 are marginal at best so not all may be real), Böttcher (2000) found that the progenitors of these bursts may need to have metallicities in excess of solar. However, the winds of collapsar progenitors must be lower than typical winds of massive stars, and hence collapsar progenitors are likely to be low-metallicity and it is unlikely that collapsars can explain such high iron abundances.

To compare the He-merger model to that of the collapsar, let us review the formation scenario of He-merger GRBs. The progenitor of this GRB engine is a binary system consisting of two massive stars. The more massive star (primary) collapses to form a neutron star or black hole. The loss of matter in the supernova explosion and a possible kick on the compact remnant unbinds many of the binaries. Those that remain bound tend to be in tight orbits, and when the companion star evolves off the main sequence, it engulfs the compact remnant and the system goes into a common envelope phase. Once in a common envelope, the compact remnant spirals down through the hydrogen star. If the binding energy of the envelope is too large, the compact star will spiral into the companion's helium core. It is this inspiral which spins up the helium core and produces the conditions necessary to form a gamma-ray burst.

The merger of the compact remnant with its helium companion provides a mechanism to produce BHAD system very similar to that of a collapsar (Fryer \& Woosley 1998). The primary difficulty with the collapsar model is producing a black hole with a rotating stellar envelope. The helium merger model naturally forms an accreting black hole surrounded by a rotating stellar core. The differences in the formation processes of these two GRB models produce distinguishing features which can be observed.

For example, after the formation of the compact remnant, the He-merger binary system will have some net velocity with respect to its formation region, and it may travel beyond the wind and supernova ejecta of the primary. Hence, the constant density environment required by some of the lightcurves is easily explained by He-mergers. In addition, the primary supernova's explosion may enrich the hydrogen envelope of its companion (Israelian et al. 1999). When this envelope is ejected during the common envelope phase, it produces an iron rich torus which may explain the observed iron lines (Böttcher \& Fryer 2000).

Fryer \& Woosley (1998) argued that the engine driving He-merger GRBs would be very similar to collapsars. But to truly understand the He-merger engine, we must follow the inspiral and obtain quantitative estimates of both the rotation of the helium core and the remnant mass in the core. Knowing these values, we can then infer the duration and total energy of the He-merger GRB.

\footnotetext{
${ }^{2}$ However, bear in mind that collapsars form only with stars that had very low mass-loss rates (presumably low metallicities) and the winds of collapsar progenitors may not alter the environment significantly (Fryer et al. 1999a). Even those GRBs which are best matched by constant density environments may yet be collapsars.
} 
In this paper, we present hydrodynamical simulations of the inspiral of a compact remnant into a helium core for a range of helium core masses. We describe our numerical techniques in $\S 2$ and the results of our simulations in $\S 3$. We conclude with a discussion of the inspiral results and of the viability of He-mergers as a GRB model.

\section{Numerical Methods}

He-merger GRBs are formed when, in a binary system, a giant star envelops its compact remnant (neutron star or black hole) companion, causing it to spiral through the hydrogen envelope in what is termed a "common-envelope" phase. The details of common envelope evolution are still rather uncertain (see Sandquist et al. 1998 for a review), but in those systems where the orbital energy of the binary is not sufficient to eject the hydrogen envelope, the helium core of the giant and the compact remnant will merge. Although it is difficult to separate the numerics from the physics in modeling the common envelope phase, modeling the merger of the helium core (which is several orders of magnitude smaller than the hydrogen giant) with a compact remnant is much more tractable.

We model the merger using the three-dimensional smooth particle hydrodynamics (SPH) code developed by Davies, Benz, \& Hills (1991). To model the physics of the merging process more accurately, the code has been improved (Fryer et al. 1999b) to include an equation of state by Blinnikov, Dunina-Barkovskaya, \& Nadyozhin (1996). To follow nuclear reactions, we include a nuclear reaction network for temperatures above $4 \times 10^{8} \mathrm{~K}$ (Timmes, Hoffman, \& Woosley 2000). The structure of the helium core was taken from 1-dimensional helium star models by Heger (1999) which were mapped into 3-dimension and then allowed to settle into a stable configuration. For all of our helium cores, the radius settled to within $10 \%$ of the 1-dimensional model.

Once the helium core has stabilized, we add the compact remnant (a point gravity source) and place the binary in a co-rotating circular orbit with a sufficiently small separation so that Roche-lobe overflow will occur. At this point, mass transfer from the helium core onto the compact remnant will tighten the orbit very rapidly and cause the compact remnant to merge with the helium core. The orbital separation at which Roche-lobe overflow commences is given by (Eggleton 1983):

$$
A_{0}=R_{\mathrm{He}} \frac{0.6 q^{2 / 3}+\ln \left(1+q^{1 / 3}\right)}{0.49 q^{2 / 3}},
$$

where $R_{\mathrm{He}}$ is the radius of the helium core, $q=M_{\mathrm{He}} / M_{\mathrm{BH}}$ is ratio of the mass of the helium core $M_{\mathrm{He}}$ to the mass of the compact remnant $M_{\mathrm{BH}}$. For our simulations, we are concerned primarily with the effects of the inspiral on the core of the helium star and these results are insensitive to our initial separation of the binary (that is, we can decrease the separation by $\sim 20 \%$ and our results do not change). For most of our simulations, we use initial separations smaller than $A_{0}$ so that the objects merge without detailed resolution of the outer layers of the helium star. In addition, it is likely that the hydrogen common envelope phase would cause the compact remnant to spiral in 
below the $A_{0}$, so using the smaller separations may even be closer to the initial conditions produced by nature. In any event, our simulations show that two models with different initial separation have similar results (Fig. 1).

Aside from initial separations, we must also be cautious with two other numerical uncertainties. First and foremost is the accretion onto the compact remnant. As the compact remnant merges with the helium star, the accretion rate onto the compact remnant is well approximated by the Bondi-Hoyle accretion rate (Chevalier 1993; Brown 1995; Fryer, Benz, \& Herant 1996; Bethe \& Brown 1998). One might argue that if the compact remnant is a neutron star, photon pressure can slow the accretion. However, the high densities in the helium core trap the photons and the time required for the photons produced at the hot surface of the neutron star to diffuse even $10 \mathrm{~km}$ from the surface would take longer than our entire simulation time (see Fryer et al. 1996 for diffusion times). However, the neutron star surface is hot enough to produce copious neutrinos which can cool the accreting material in less than $0.1 \mathrm{~s}$ and this cooling (which however does not provide pressure support as photons would) allows the infalling material to accrete onto the neutron star (see Fryer et al. 1996). Chevalier (1989) argued that even if the compact remnant had dipole magnetic fields in excess of $10^{15} \mathrm{G}$, these fields would be buried by the high accretion flows that occur in He-mergers. The more conservative analysis of Fryer et al. (1996) argues that the compact remnant's magnetic field would have to exceed $10^{13} \mathrm{G}$ to affect the accretion. Such highly magnetized neutron stars are probably not common. Hence, for neutron star and black hole remnants alike, the inspiralling compact remnant will accrete at the Bondi-Hoyle rate. However, actually modelling this accretion can be quite difficult.

We try two methods to simulate the hypercritical accretion. In the first accretion recipe, we simply remove particles that fall within $5-10 \times 10^{8} \mathrm{~cm}$ of the compact remnant and add their mass and momentum to the compact remnant. Although this is much larger than the Schwarzschild radius of the compact remnant, it is the smallest scale we can achieve given our resolution (1-3 $\left.\times 10^{9} \mathrm{~cm}\right)$.

In the second accretion method, when the distance between a particle and the compact remnant, $r_{\mathrm{p}}$, is less than the scale length $h$ of that particle, the mass of that particle $M_{\mathrm{p}}$ is accreted onto the compact remnant with a rate given by $\dot{M}_{\mathrm{p}}=M_{p} / t_{\mathrm{ff}}$, here $t_{\mathrm{ff}}$, the free-fall timescale is $\sqrt{2 r_{\mathrm{p}}^{3} / G M_{\mathrm{BH}}}$, where $G$ is the gravitational constant and $M_{\mathrm{BH}}$ is the compact remnant mass. In this method, if its distance from the black hole is less than $h$, the particle's motion is dominated by gravitational force of the compact remnant and it loses mass on the free-fall timescale. We reduce the particle's mass gradually as it accretes onto the compact remnant. The second accretion method yields a slightly longer inspiral time (by 10-20\%: see Fig. 1) which, in turn, yields accretion masses which differ by roughly the same amount (see $\S 3$ ). To test the differences in the accretion rate, we ran a test model where we placed a $2 M_{\odot}$ compact remnant in the center of an $\sim 8 M_{\odot}$ static helium core and measured the accretion as a function of time (Fig. 2). Here we see that the accretion rates for both our accretion mechanisms are nearly identical. 
One might argue that the accretion timescale is not the free-fall timescale because angular momentum can slow the accretion. However, during the inspiral stage of the merger of a helium and a compact remnant, the angular momentum of particles accreted by the compact remnant is small. For the duration of our simulation, the accretion timescale is dominated by the free-fall timescale, not by the viscous timescale of the accretion disk. However, to test the differences caused by an increased accretion timescale due to the additional effects of a viscous timescale, we modified the second accretion method by assuming the accretion timescale was 10 times the free-fall timescale and ran our test model with this increased timescale (Fig. 2). The accretion rate for this factor of 10 range in timescales is nearly identical for the first $150 \mathrm{~s}$ and does not differ by more than $20 \%$ for the entire simulation.

This is not a surprising result. By increasing the accretion timescale, we initially lower the accretion rate onto the black hole, but this does not lower the rate at which material piles up around the black hole (which is what our hydrodynamic simulation is modeling). When we raise the accretion timescale, more material piles up around the black hole, and the density of this material increases, causing the accretion rate to rise. Hence an equilibrium is achieved. The accretion rate onto the black hole is more sensitive to the rate at which the material piles up around the black hole than upon the accretion timescale of any individual particle. This is nearly identical to the effect seen in accretion disk simulations where the accretion rate from the innermost point of the disk onto a black hole is governed more by the rate at which material is put into the disk rather than the actual value of the artificial viscosity (Popham, Woosley, \& Fryer 1999; MacFadyen \& Woosley 1999). Because the accretion rate onto the black hole is relatively insensitive to our accretion recipe and because the first recipe, which simply removes nearby particles around the black hole (or neutron star), runs faster than other recipes, we use the first recipe in all other simulations.

In both methods, we also must add the angular momentum of the particles to the compact remnant. Unfortunately, we do not resolve the accretion disk which must form around the compact remnant and so cannot model the transport of angular momentum out of this disk as it accretes. Instead, we constrain the amount of angular momentum accreted to the angular momentum of the last stable orbit of the compact remnant. We assume the rest of the angular momentum is transported out of the system. Because our simulation time step is much longer than the orbital period of the last stable orbit, this is a reasonable assumption. This angular momentum may feedback into the star, and we do not include this. But the angular momentum that we discard in our simulation is less than $1 \%$ of the total angular momentum in the system (Fig. 3), so we do not underestimate the angular momentum in the helium core by more than $1 \%$ at the end of our simulation.

Figure 3 also shows how well the total angular momentum in our simulations is conserved. For the merger of a $2 M_{\odot}$ compact remnant and an $\sim 8 M_{\odot}$ helium star, the summation of total angular momentum of particles, total angular momentum of the compact remnant (including the estimated spin angular momentum) and the angular momentum that we discard during accretion is conserved to one part in ten thousand. During the course of the simulation, nearly all of the 
orbital angular momentum of the compact remnant is converted into spin angular momentum of the helium core, and yet the total angular momentum is conserved!

The other major numerical uncertainty is the artificial viscosity used in the SPH. We have varied the artificial viscosity by a factor of 5 to determine our dependence upon the numerical viscosity. We will discuss these results in $\S 3$, but the quick answer is that simulations using this wide range of artificial viscosity obtain nearly the same results and our estimates of GRB energies and disk structures do not depend on the artificial viscosity.

\section{Results}

We have run a series of models testing the initial helium star masses, initial separations, and the range of uncertainties in the numerical methods (accretion algorithm, artificial viscosity, resolution, etc.). Böttcher \& Fryer (2000) found that the helium star masses in He-merger progenitors cover a wide range of masses: $14 \%$ have masses less than $7 M_{\odot}$ and $22 \%$ have masses above $12 M_{\odot}$ with the bulk $(64 \%)$ lying within $7-12 M_{\odot}$. By varying the mass of the helium star, we can study the range of burst properties from He-mergers. The entire set of simulations is summarized in Table 1. The number in the model designation denotes the helium core mass in units of solar mass.

We break up the formation of a He-merger GRB engine into two stages: the inspiral of the compact remnant (which we model with our SPH code) and a post-processing analysis of the accretion onto the compact remnant once it reaches the core of the helium star. In our simulations, we follow the compact remnant as it spirals into the helium star and accretes mass from the helium star while spinning up the star. When the compact remnant reaches the center of the helium core,

our simulations provide the mass and spin of the compact remnant as well as the structure and angular momentum distribution of the helium star.

The results from our simulations allow us to estimate the accretion of the rotating helium core around the now central black hole. We know that this material will accrete rapidly (especially along the spin axis of the core), but the material along the equator has too much angular momentum to accrete immediately and will first form a disk around the compact remnant. It is this disk accretion that can drive a GRB along the relatively clear spin axis. The mass of the compact remnant and the spin of the accreting material determine the viability of He-mergers as a GRB engine.

\subsection{Inspiral}

We presume that the compact remnant has already spiralled through the hydrogen envelope of a massive giant star (via a common envelope phase) and begin our simulations as the helium core contacts the compact remnant in Roche lobe overflow. For two of our simulations (8D and $8 \mathrm{E}$ ) we placed the compact remnant in a circular orbit with an orbital separation just low enough 
that the helium star overfilled its Roche lobe and accreted onto the compact remnant. Even if orbital angular momentum were conserved, this mass transfer (from a more massive object onto a less massive object) would tighten the orbits. But, in fact, a good deal of angular momentum is lost as material is ejected from the system. In these simulations, we have implicitly assumed that when Roche-lobe overflow occurs, this mass transfer dominates the orbital evolution of the binary.

The viscosity caused by the hydrogen envelope may dominate the inspiral even after the helium core has overfilled its Roche lobe and is accreting onto the compact remnant (although we know that when the compact remnant actually enters the helium core, the effects of the helium core must dominate the inspiral). Fortunately, most of the accretion occurs after the compact remnant has spiralled deep into the helium star and the merger evolution below a separation of $\sim 5 \times 10^{10} \mathrm{~cm}$ is not affected by our initial separation (Fig. 1). Thus, for our $16 M_{\odot}$ and $4 M_{\odot}$ helium cores, we only model the inner $\sim 70 \%$ of the core and start our simulations with smaller separations. This allows us to better resolve the core with a given number of particles.

Some snapshots of the inspiral process for models $8 \mathrm{~B}, 4 \mathrm{~A}$ and $16 \mathrm{~A}$ are shown, respectively, in Figures 4, 5 and 6. Some material is ejected, carrying away angular momentum and some mass. In general, this ejecta does not play an important role on the GRB explosion itself because it is likely that the GRB explosion is beamed along the axis of rotation and the ejecta lies along the equator. But this ejecta may effect the environment around the GRB and may have some observational features (e.g. iron lines - Böttcher \& Fryer 2000).

Although the binary can survive many orbital periods in the Roche-lobe mass-transfer phase, as soon as the compact remnant begins to merge with the helium core, the orbital separation evolves very rapidly. The compact remnant inspirals from half its initial separation to the center of the helium star in less than $\sim 500 \mathrm{~s}$ (Fig. 7). It is during this latter evolution that most of the accretion onto the compact remnant occurs and the conditions for a GRB engine are produced. Changing the artificial viscosity by a factor of 5 changes this inspiral time by less than $10 \%$. Also, results of high resolution model $8 \mathrm{~B}$ are similar to those of low resolution model 8A (Fig. 7).

In the initial stages of the inspiral, the accretion rate onto the compact remnant is relatively low (less than $10^{-4} M_{\odot} \mathrm{s}^{-1}$ ) and the compact remnant gains very little mass. But during the rapid inspiral phase, the accretion rate increases dramatically and nearly all of the accretion occurs in the last few hundred seconds. We stop our simulations when the compact remnant reaches the "center" of the helium star.

After the inspiral stage, the effect of angular momentum which can slow the accretion gradually becomes important. However, at the end of our simulations, the effect of angular momentum is still small. For example, at the end of the simulation for model $8 \mathrm{~B}$, the viscous timescale of most of the matter (93\% of particles) accreting onto the black hole is less than the free-fall timescale (we estimate the viscous timescale by: $t_{\text {visc }}=(\alpha \omega)^{-1}$ where $\alpha=0.1$ is the disk viscosity and $\omega$ is the orbital angular velocity). However, the effect of angular momentum is increasing rapidly, and if we want to model the accretion beyond the inspiral phase, we would need to modify our accretion 
recipe in addition to increasing our mass resolution.

The mass of the compact remnant at the end of our simulations are given in Table 2. At this stage, nearly all of the orbital angular momentum of the system has been converted to spin angular momentum in the helium star (Fig. 8). Some of the orbital angular momentum is converted into spin of the compact remnant (Table 2). This merged system is now very similar to that of a collapsar (a rotating helium star collapsing onto a central black hole). These conditions are believed to drive GRB explosions, but to estimate the explosion energy, we must calculate the later accretion.

\subsection{Accretion Rate}

Our simulations show that He-mergers will indeed produce rapidly rotating helium stars accreting onto a central compact remnant in much the same manner as is found in the collapsar GRB engine. However, the angular momentum in He-mergers (Fig. 8) is roughly an order of magnitude greater than that of the collapsars studied by MacFadyen \& Woosley (1999). With such high angular momentum, following the continued accretion requires an understanding of disk accretion. Even if we assumed an artificial angular momentum transport algorithm, we would need much more resolution than we have in our current simulations to follow disk formation and the accretion onto the compact remnant.

However, we can analytically estimate the accretion rate onto the compact remnant by estimating the accretion time for each particle. Such a rough calculation can be obtained by assuming that pressure support is negligible and gravitational and centrifugal forces dominate the motion of the particles (for most of the star, this is true).

We take the end of our SPH simulations as the initial conditions of our analytic estimate. Without pressure support, each particle accelerates to nearly the free-fall velocity until centrifugal support slows its collapse and it accretes onto a disk. We can derive the radius at which this occurs by knowing the enclosed mass $\left(M_{\text {Enclosed }}\right)$ and the angular momentum $(j)$, and then equating forces:

$r_{\text {disk }}=j^{2} / G M_{\text {Enclosed }}$. We estimate the timescale for the particle to accrete onto the disk is simply the free-fall timescale:

$$
t_{\mathrm{ff}}=\sqrt{\frac{2 r_{\text {initial }}^{3}}{G M_{\text {enclosed }}}}-\sqrt{\frac{2 r_{\text {disk }}^{3}}{G M_{\text {enclosed }}}},
$$

where $r_{\text {initial }}$ is the initial position of the particle. For matter along the pole, the angular momentum may not be sufficient to halt the collapse, and these particles accrete directly onto the compact remnant at roughly the free-fall time.

But the bulk of the matter will accrete through a disk, and we must then estimate the disk accretion timescale. For each particle in the disk, we can calculate the viscous timescale:

$$
t_{\mathrm{visc}}=(\alpha \omega)^{-1}
$$


where $\alpha$ is the disk viscosity parameter (which we take to be 0.1), and $\omega$ is the orbit angular velocity. The total accretion timescale of each particle is just the sum of its free-fall and viscous components $\left(t_{\mathrm{acc}}=t_{\mathrm{ff}}+t_{\mathrm{visc}}\right)$. We can then easily estimate the accretion rate by calculating the number (and total mass) of particles accreted at a given time $\left(t_{0}\right)$

$$
\dot{M}=\sum_{t_{0}-\Delta t<t_{\mathrm{acc}}^{i}<t_{0}+\Delta t}^{i} \frac{m_{i}}{2 \Delta t},
$$

where $m_{i}$ and $t_{\mathrm{acc}}^{i}$ are the mass and accretion rate of particle $i$. The accretion rates for our 3 helium star masses are shown in Figures 9, 10 and 11. The accretion rate not only provides us with an estimate of the black hole mass as a function of time, but also the spin of the black hole. There is so much angular momentum in the helium core that nearly all of the accreting material accretes through a disk, and the angular momentum of the material at its last stable orbit quickly spins up the black hole.

In Figures 9 - 11, the accretion rates seem to vary on short timescales, but such wide variations are likely to be an artifact of our low resolution. Higher resolution simulations will allow us to determine how much of the variation in the accretion rate is real (due to asymmetries caused in the inspiral) and how much is due to numerical resolution.

Throughout our calculation, we have tried to estimate an upper limit to the accretion rate, assuming no thermal pressure and a high disk viscosity. Even so, except for the $16 M_{\odot}$ merger, the accretion rates we obtain are much lower than those calculated in collapsars. This is, in part, because our helium cores have much higher angular momenta. In addition, we modeled the merger of unevolved helium cores. If the common envelope phase occurred during Case C mass-transfer, the merger would not have occurred until after helium burning, and the more compact evolved core would yield higher initial accretion rates.

\section{Gamma-Ray Bursts}

In this paper, we present the first hydrodynamical simulations of the formation of He-merger GRBs up to the formation of a central black hole around a rotating helium core. With the conditions produced during the inspiral, it is clear that the compact remnant will collapse to a black hole (if it is not one already). The densities around the central compact remnant are so high that photons are trapped in the material and radiation pressure does not halt accretion. Even if the compact remnant is a neutron star, neutrino cooling will allow high accretion rates $\left(>10^{5} M_{\odot} \mathrm{yr}^{-1}\right)$ which will quickly cause the neutron star to collapse to a black hole. The accreting material will also spin up the compact remnant and He-mergers produce a population of rapidly rotating black holes. With

possible merger rates above $10^{-5} \mathrm{yr}^{-1}$ in the Galaxy, these black holes may make up a few percent of the total black holes formed in the galaxy. However, they do not produce slowly accreting neutron stars (e.g. Thorne-Zytkow stars) and cannot be used to explain strange neutron star systems. 
But can these mergers produce gamma-ray bursts? The viability of the He-merger GRB model depends upon the energy of the explosion produced during the disk accretion phase. The two mostoften discussed power sources for these explosions are: neutrino annihilation or some magnetic field mechanism akin to the engine behind the jets of active galactic nuclei. The neutrino annihilation mechanism takes advantage of the profuse neutrino emission which carries off much of the binding energy of the accreting material. Popham et al. (1999) calculated the energy emitted in neutrinos for hyper-accreting black holes. The subsequent energy generation rate from neutrino annihilation can be fitted by (Fryer et al. 1999b):

$$
\log L_{\nu, \bar{\nu}} \operatorname{ergs} s^{-1} \approx 43.6+4.89 \log \frac{\dot{M}}{0.01 M_{\odot} \mathrm{s}^{-1}}+3.4 a,
$$

where $a \equiv J_{\mathrm{BH}} c / G M_{\mathrm{BH}}^{2}$ is the spin parameter of the black hole. Using this formula, and our derived accretion rates, black hole spin evolution, etc., we can estimate the energy deposition rate $\left(L_{\nu, \bar{\nu}}\right)$ for helium mergers of different initial helium star mass (Fig. 9-11). We stress that the variation in the energy deposition is almost certainly due to our low numerical resolution, and it will require much higher resolution simulations which can resolve any clumping structures to determine what fluctuations, if any, are produced in the accretion. Integrating $L_{\nu, \bar{\nu}}$, we get the estimates of the total explosion energy (Table 2). With beaming into $3 \%$ of the sky, Model 16A produces an equivalent isotropic energy of $10^{54} \mathrm{ergs}$ ! However, we should bear in mind that there are many uncertainties in these calculations: variations in the numerics (compare models 8A, 8B, $8 \mathrm{C})$, simplified assumptions in our accretion rates, and the uncertainties in the neutrino annihilation deposition. The errors in our calculations of the explosion energy may exceed 1 order of magnitude. Because we chose high disk viscosities and assumed none of the energy produced would be dragged back into the accreting remnant, our estimates are likely to be upper limits to the burst energies. Even so, except for He-mergers involving massive helium cores $\left(\sim 16 M_{\odot}\right)$, helium mergers powered by neutrino annihilation cannot produce the most energetic GRBs. However, $22 \%$ of He-mergers involve the merger of compact remnants and massive helium stars $\left(>12 M_{\odot}\right.$ : Böttcher \& Fryer 2000) and the rate of massive helium-star mergers may well be high enough to explain the observed rate of GRBs.

More promising for the low accretion rates in He-mergers, MHD processes may extract the rotational energy of either the black hole or the accretion disk and power the GRB (Blandford \& Znajek 1977; MacDonald et al 1986; Paczyński 1991; Narayan, Paczyński, \& Piran 1992; Woosley 1993; Hartmann \& Woosley 1995; Thompson 1996; Katz 1997; Mészáros \& Rees 1997; Popham et al. 1999). For example, the Blandford-Znajek luminosity is

$$
L_{\mathrm{BZ}} \approx 10^{50} a^{2}\left(\frac{M_{\mathrm{BH}}}{3 M_{\odot}}\right)^{2}\left(\frac{B}{10^{15} \mathrm{Gauss}}\right)^{2} \operatorname{ergs~}^{-1},
$$

where $a$ is the spin parameter of the black hole and $B$ is the magnetic field strength in the disk. We know the spin rate $(a)$ and mass $\left(M_{\mathrm{BH}}\right)$ of the black hole and we can get an rough upper limit to the magnetic field strength by setting the magnetic energy density equal to the kinetic energy 
density of accreting disk. This yields:

$$
B^{2}=4 \pi \rho v^{2} \approx \dot{M} c / r_{\mathrm{g}}^{2}
$$

where $r_{\mathrm{g}} \equiv 2 G M / c^{2}$. Then we can estimate the maximum energy deposition rate for explosions driven by magnetic fields (Fig. 12).

When an explosion is produced, it can disrupt the disk and halt further accretion. This will turn off the engine. We assume the deposition continues for $200 \mathrm{~s}$ (a long burst) and integrate the deposition rate to calculate maximum burst energies (Table 2). Burst energies above $10^{51}$ ergs are possible for all our He-merger models. These energies are most certainly overestimates, but lacking a working magnetic field mechanism, reliable calculations (with errors less than a few orders of magnitude) are impossible.

However, it is clear that He-mergers can produce GRB explosions. Even with neutrino annihilation as the energy source, mergers of massive helium stars and compact remnants can explain long-duration bursts with inferred isotropic energies of above $10^{54} \mathrm{ergs}$. Because He-mergers are similar to collapsars (with slightly higher specific angular momenta), we believe they will have very similar characteristics to collapsars: they are likely to be beamed, and will produce supernovae-like explosions (e.g. 1998bw).

This research has been supported by NASA (NAG5-2843, MIT SC A292701, and NAG5-8128), the NSF (AST-97-31569), the US DOE ASCI Program (W-7405-ENG-48) and the Los Alamos Feynman Fellowship. The authors gratefully appreciate stimulating conversations with Andrew MacFadyen and Stan Woosley on the subject of gamma-ray bursts and Collapsars. We thank Stan Woosley and Tamara Rogers for helpful comments on the manuscript.

\section{REFERENCES}

Belczyński, K., Bulik, T., \& Rudak, W. 2000, Proc. of the 5th Huntsville Gamma-Ray Burst Symposium, Oct. 1999, ed. R.M. Kippen, AIP

Bethe, H.A., \& Brown, G.E. 1998, ApJ, 506, 780

Blandford, R.D., \& Znajek, R.L. 1977, MNRAS, 179, 433

Blinnikov, S.I., Dunina-Barkovskaya, N.V., \& Nadyozhin, D.K. 1996, ApJS, 106,171

Böttcher, M., 2000, ApJ, 539, 102

Böttcher, M., \& Fryer, C.L. 2000, in preparation

Brown, G.E. 1995, ApJ, 440, 270 
Chevalier, R.A. 1989, ApJ, 346, 847

Chevalier, R.A. 1993, ApJ, 411, L33

Chevalier, R.A., \& Li, Z.-Y. 2000, ApJ, 536, 195

Davies, M.B., Benz, W., \& Hills, J.G. 1991, ApJ, 381, 449

Eggleton, P.P. 1983, ApJ, 268, 368

Fryer, C.L., Benz, W., \& Herant, M. 1996, ApJ, 460, 801

Fryer, C.L., Woosley, S.E. 1998, ApJ, 502, L9

Fryer, C.L., Woosley, S.E., \& Hartmann, D.H. 1999a, ApJ, 526, 152

Fryer, C.L, Woosley, S.E., Herant, M., \& Davies, M.B. 1999b, 520, 650

Hartmann, D.H., \& Woosley, S.E. 1995, Adv. Space Res., 15 (5), 143

Heger, A. 1999, Private Communication

Israelian, G., Rebolo, R., Basri, G., Casares, J., \& Martin, E.L. 1999, Nature, 401, 142

Katz, J.I. 1997, ApJ, 490, 633

Katz, J.I., \& Canel, L.M. 1996, in Third Huntsville Gamma-Ray Burst Symp., ed. C.Kouveliotou, M.F. Briggs \& G.J. Fishman (Woodbury: AIP), 759

Kulkarni S.R., et al. 1998, Nature, 395, 663

Kouveliotou, C., et al. 1993, ApJ, 413, L101

Livio, M., \& Waxman, E. 2000, ApJ, 538, 187

MacDonald, D.A., Thorne, K.S., Price, R.H., \& Zhang, X.-H. 1986, in Black Holes, the Membrane Paradigm, ed. K.S. Throne, R.H. Price, \& D.A. Macdonald (New Haven: Yale Univ. Press), 138

MacFadyen, A.I., \& Woosley, S.E. 1999, ApJ, 524, 262

MacFadyen, A.I., Woosley, S.E., \& Heger, A. 2000, ApJ, in press (astro-ph 9910034)

Mészáros, P. \& Rees, M.J. 1997, ApJ, 482, L29

Narayan, R., Paczyński, B., \& Piran, T. 1992, ApJ, 395, L83

Paczyński, B. 1991, Acta Astron., 41, 257

Piro, L., et al. 1999, ApJ, 514, L73 
Piro, L., et al. 2000, submitted to Science

Popham, R., Woosley, S.E., \& Fryer, C.L. 1999, ApJ, 518, 356

Sandquist, E.L., Taam, R.E., Chen, X., Bodenheimer, P., Burkert, A. 1998, ApJ, 500, 909

Tavani, M. 1998, ApJ, 497, L21

Thompson, C. 1996, in Gamma-Ray Bursts: 3rd Huntsville Symp., AIP Conf. Proc. 384, ed. C. Kouveliotou, M. Briggs, \& G. Fishman, AIP: New York, 709

Timmes, F.X., Hoffman, R.D., \& Woosley, S.E. 2000, ApJ, in press

Woosley, S.E. 1993, ApJ, 405, 273

Yoshida, A., et al. 1999, A\&AS, 138, 433 
Table 1. Parameters of Models

\begin{tabular}{ccccccc}
\hline \hline Model & $\begin{array}{c}M_{\text {Remnant }} \\
\left(M_{\odot}\right)\end{array}$ & $\begin{array}{c}M_{\text {He }} \\
\left(M_{\odot}\right)\end{array}$ & $\begin{array}{c}\text { Initial } \\
\text { Separation } \\
\left(10^{8} \mathrm{~cm}\right)\end{array}$ & $\begin{array}{c}\text { \# of } \\
\text { Particles }\end{array}$ & $\begin{array}{c}\text { Accretion } \\
\text { Recipe }\end{array}$ & $\begin{array}{c}\text { Artificial } \\
\text { Viscosity }\end{array}$ \\
\hline 8A & 2 & 8 & 1000 & 10000 & First & High \\
8B & 2 & 8 & 1000 & 30000 & First & High \\
8C & 2 & 8 & 1000 & 10000 & First & Low \\
8D & 2 & 8 & 1200 & 10000 & First & High \\
8E & 2 & 8 & 1200 & 10000 & Second & High \\
4A & 2 & 4 & 650 & 10000 & First & High \\
16A & 2 & 16 & 575 & 10000 & First & High \\
\hline
\end{tabular}


Table 2. Energy \& Duration

\begin{tabular}{cccccc}
\hline \hline Model & $\begin{array}{c}M_{i}{ }^{\mathrm{a}} \\
\left(M_{\odot}\right)\end{array}$ & $a_{i}{ }^{\mathrm{a}}$ & $\begin{array}{c}E_{\nu, \bar{\nu}}^{\mathrm{b}} \\
\left(10^{49} \mathrm{ergs}\right)\end{array}$ & $\begin{array}{c}T_{90}^{\mathrm{burstc}} \\
(\mathrm{s})\end{array}$ & $\begin{array}{c}E_{\mathrm{BZ}}{ }^{\mathrm{d}} \\
\left(10^{49} \mathrm{ergs}\right)\end{array}$ \\
\hline 8A & 2.25 & 0.14 & 0.51 & 370 & $<630$ \\
8B & 2.87 & 0.60 & 0.049 & 510 & $<480$ \\
8C & 2.76 & 0.43 & 0.090 & 520 & $<500$ \\
4A & 2.15 & 0.21 & 0.018 & 460 & $<220$ \\
$16 \mathrm{~A}$ & 5.54 & 0.985 & 3700 & 65 & $<5500$ \\
& & & & & \\
\hline
\end{tabular}

${ }^{a}$ The mass and spin of the compact remnant when it reaches the center of the helium star.

${ }^{\mathrm{b}}$ These are maximum total energies emitted via neutrino annihilation. We assume no energy is carried in along the poles.

${ }^{\mathrm{c}}$ The burst duration for neutrino annihilation driven explosions is the timescale in which $90 \%$ of the total energy is emitted.

dThese are probably maximum total energies from a magnetically driven mechanism, assuming that the magnetic field energy density equals the kinetic energy of the accreting disk. 


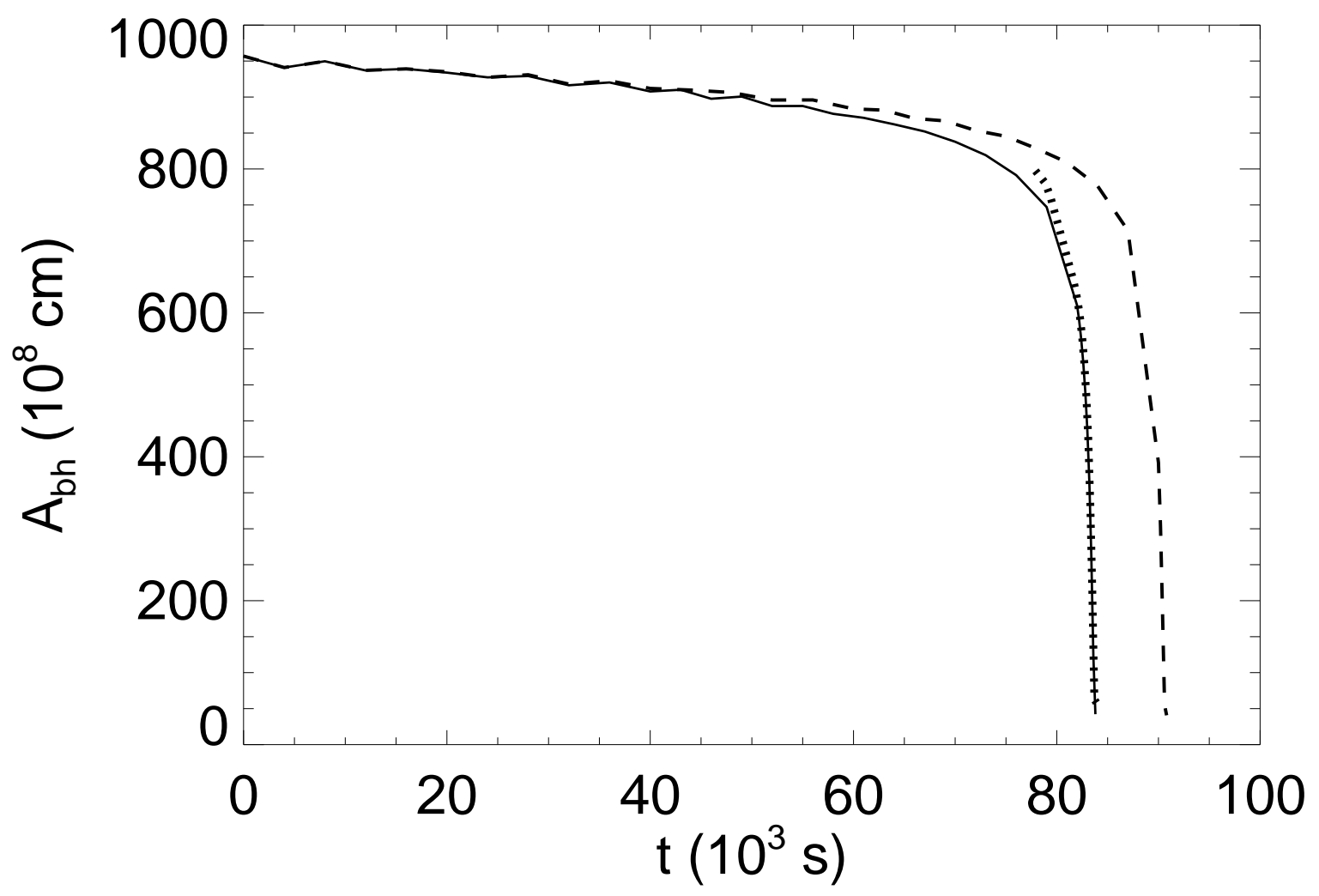

Fig. 1. - The distance between the compact remnant and the center of the mass vs. time. The solid line show results with large initial separation for our first accretion recipe, which simply removes nearby particles around the compact remnant. The dotted line show results with small initial separation for the first accretion recipe. The beginning of dotted line is at $80000 \mathrm{~s}$. The dotted line has been shifted along the time axis to compare the effects of our initial separation on the late-time inspiral. Note that the inspiral in the core is nearly identical for both initial separations. The dashed line show results with large initial separation for our second accretion recipe, which gradually removes the mass from particles as they accrete onto the nearby compact remnant. 


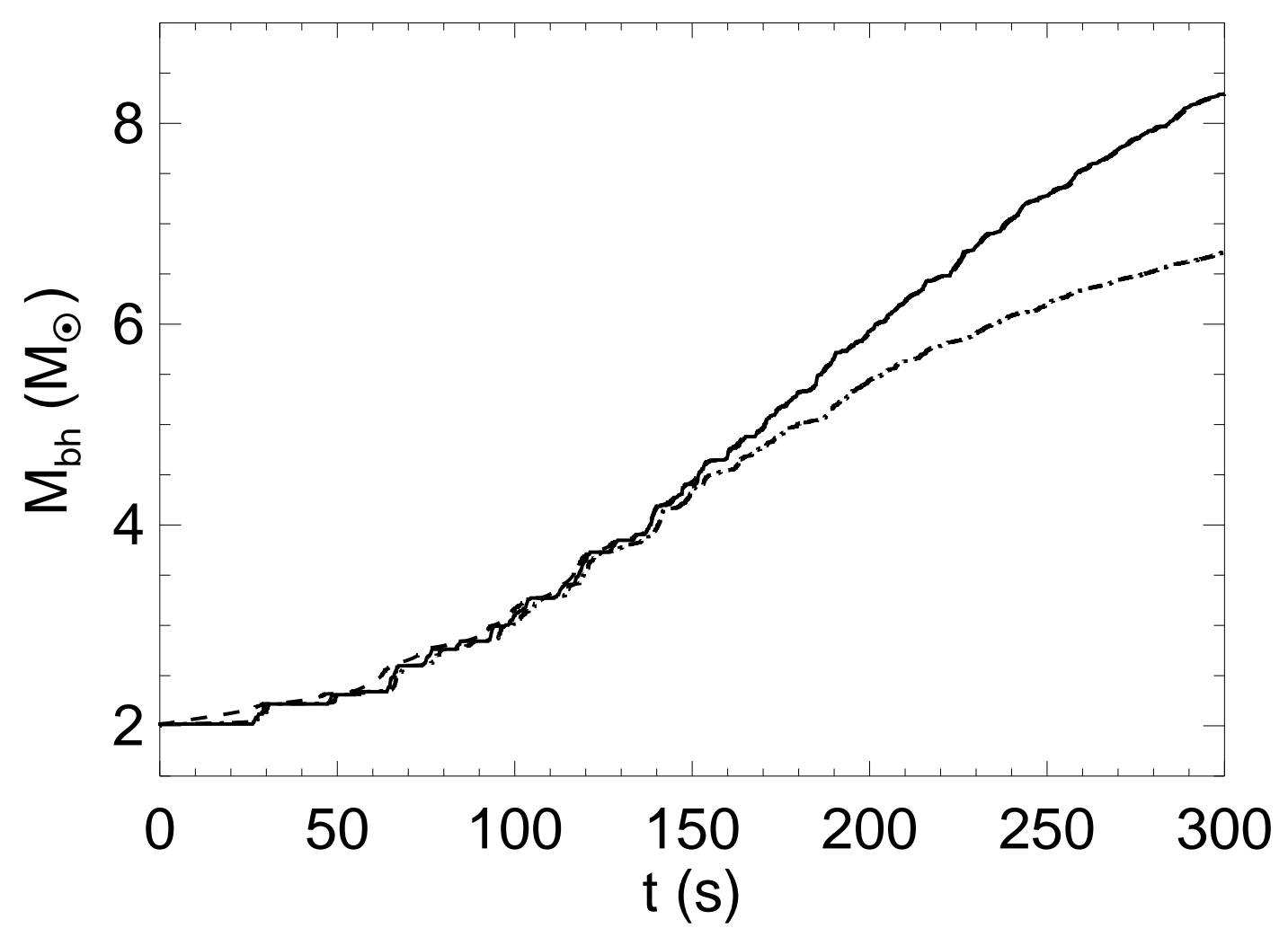

Fig. 2.- The mass of the compact remnant vs. time for a central object accreting an $8 M_{\odot}$ helium star. The solid line shows results for the first recipe, which simply removes nearby particles around the compact remnant, the dashed line shows results for the second recipe, which gradually removes the mass from particles as they accrete onto the nearby compact remnant. The accretion rate for this second recipe is determined by the free-fall timescale. As the angular momentum of the accreting material increases, the viscous timescale necessary to remove this angular momentum can dominate the accretion timescale. The dash dot line shows results for a variation of the second recipe, where we assume that the accretion timescale is 10 times the free-fall timescale (assuming that the viscous timescale is much larger than the free-fall timescale). Because the particles do not accrete as quickly, they build up around the compact remnant, creating higher densities and, hence, shorter accretion timescales and the total accretion rate only changes $10-20 \%$ even though we decreased the timescale by a factor of 10 ! 


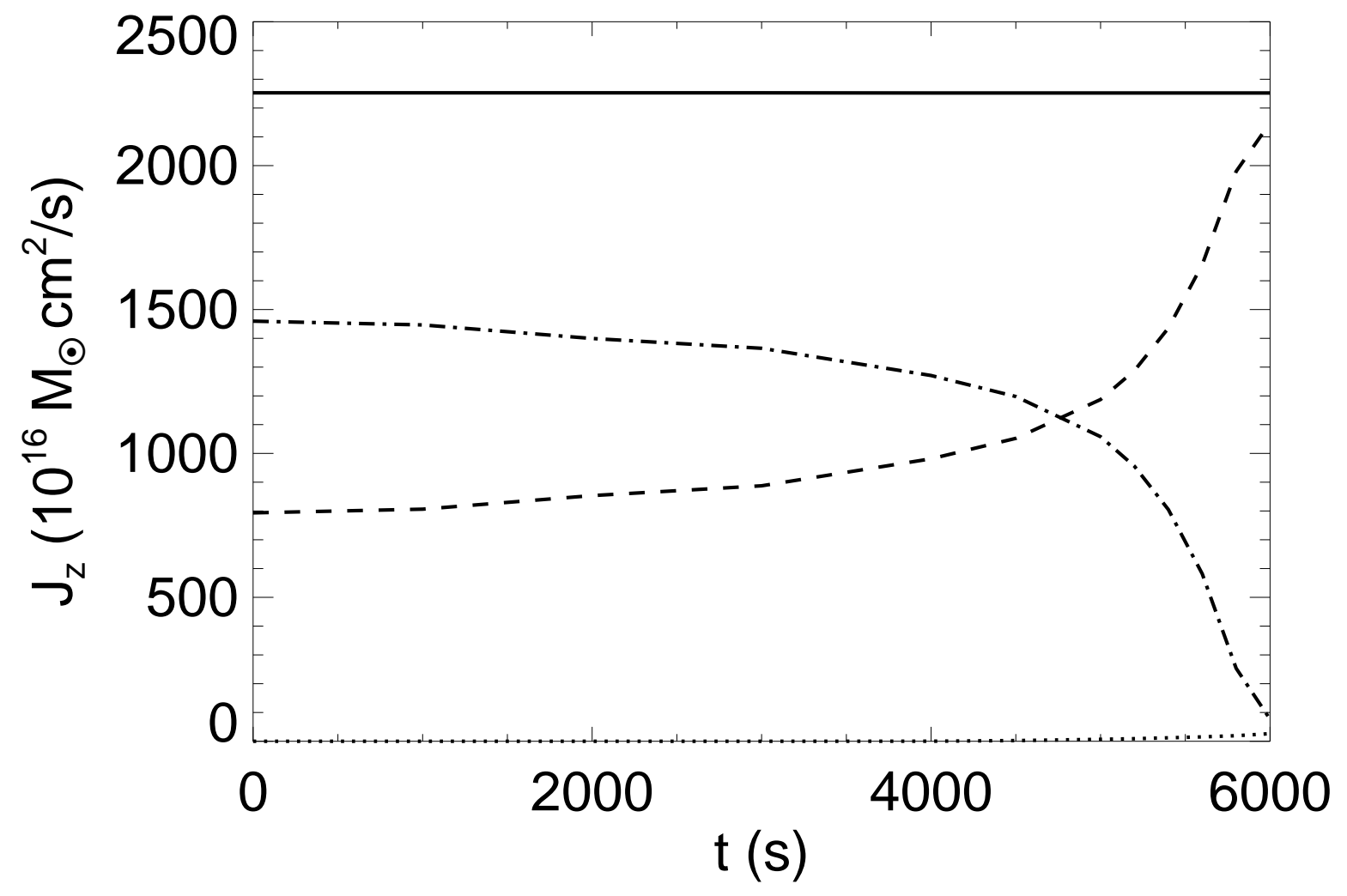

Fig. 3.- The angular momentum vs. time. The solid line denotes total angular momentum, the dashed line denotes angular momentum of the helium core, the dash dot lines denotes angular momentum of the compact remnant, and the dotted line denotes the angular momentum which is not accreted in the disk and is discarded in our simulations. 


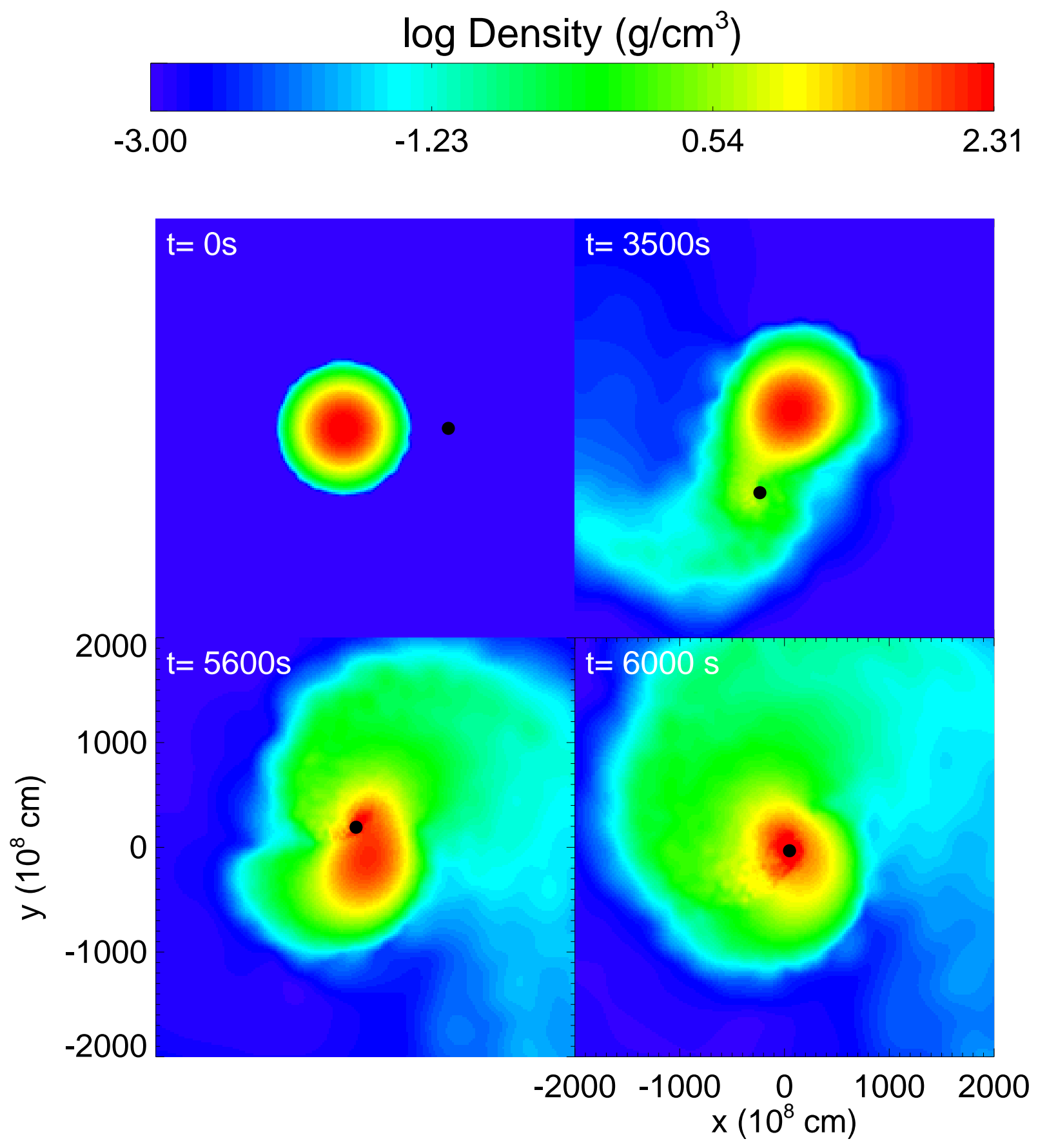

Fig. 4.- Merger of a $2 M_{\odot}$ compact remnant with a $8 M_{\odot}$ helium core. 


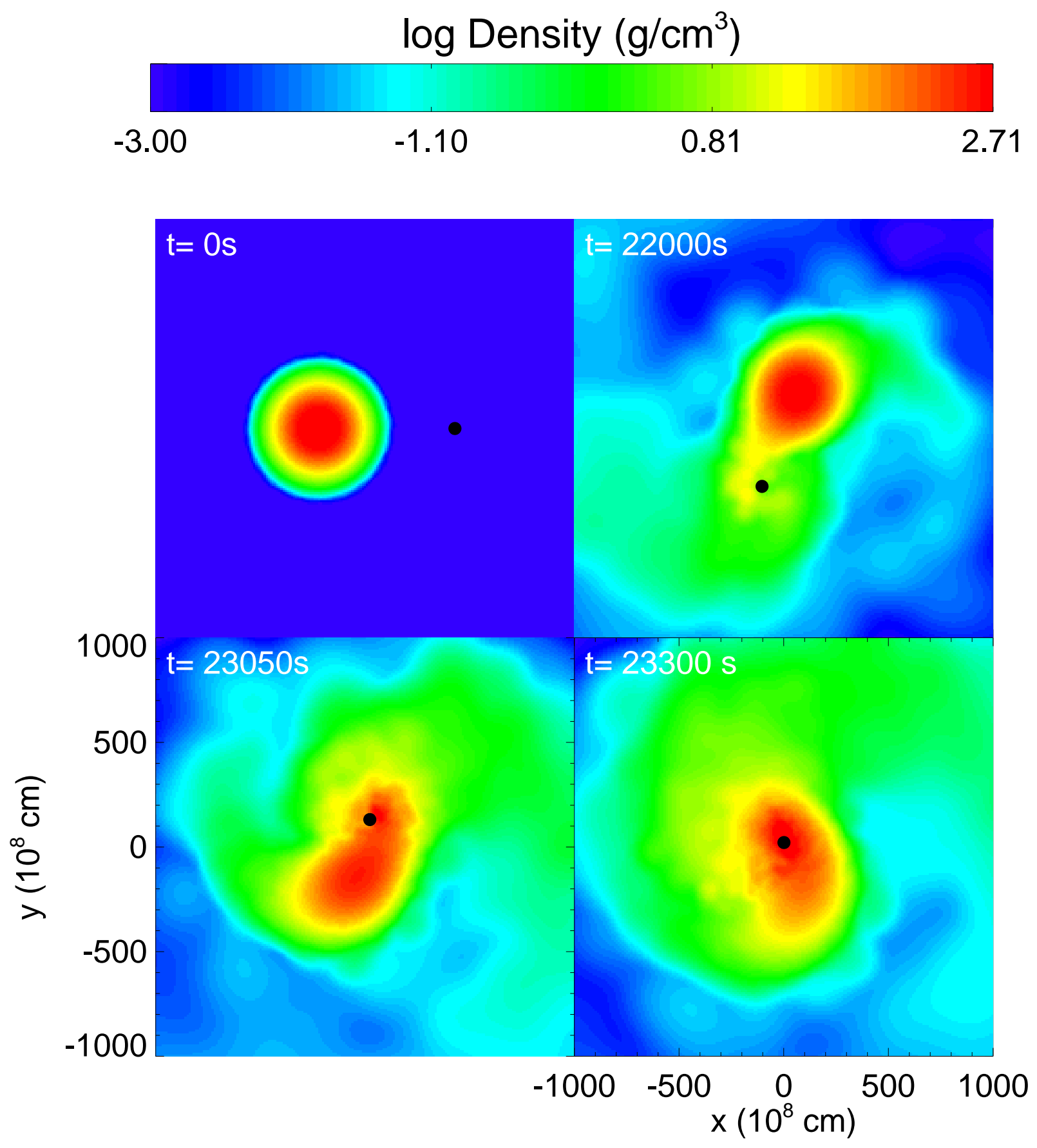

Fig. 5.- Merger of a $2 M_{\odot}$ compact remnant with a $4 M_{\odot}$ helium core. 


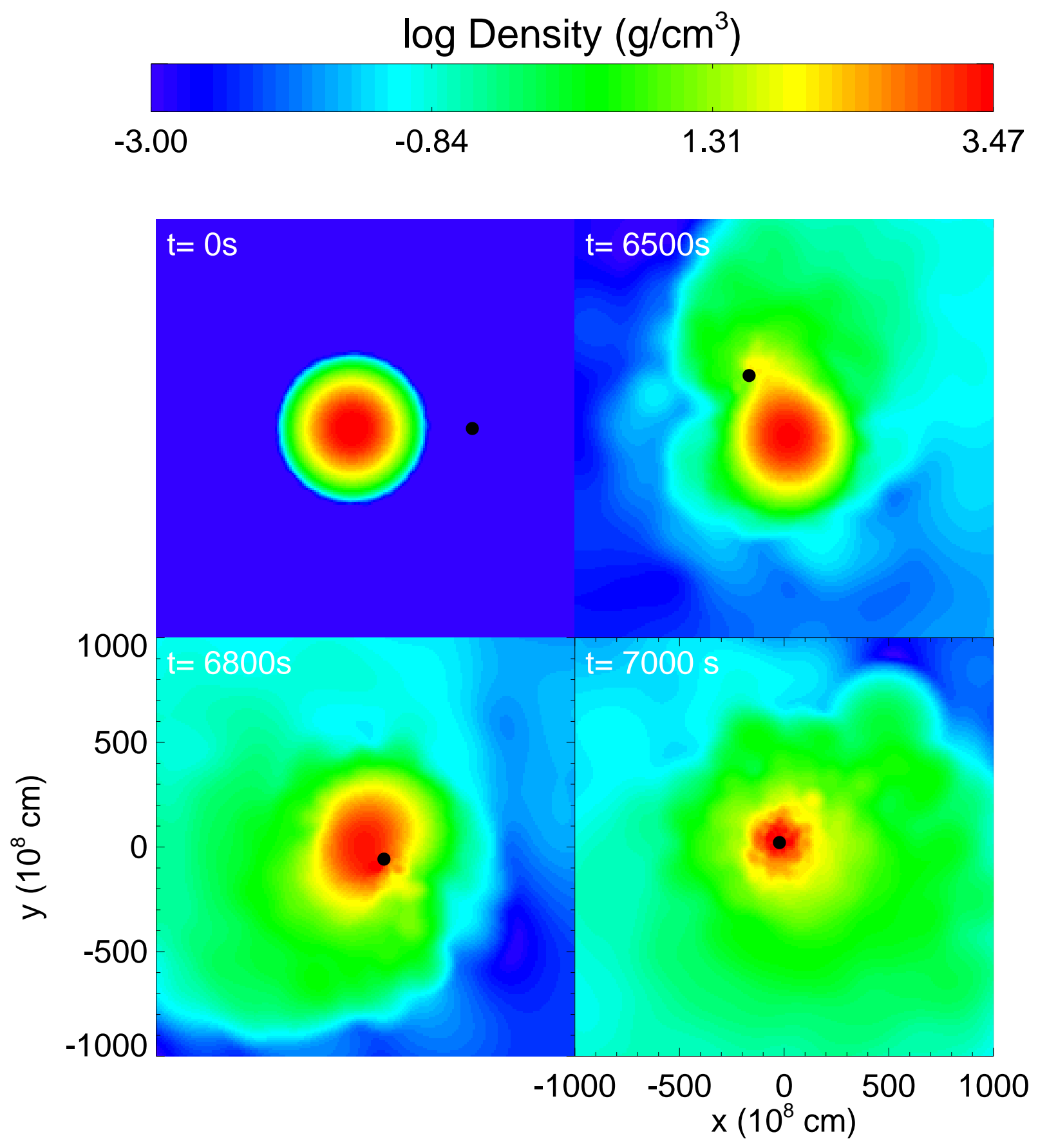

Fig. 6.- Merger of a $2 M_{\odot}$ compact remnant with a $16 M_{\odot}$ helium core. 

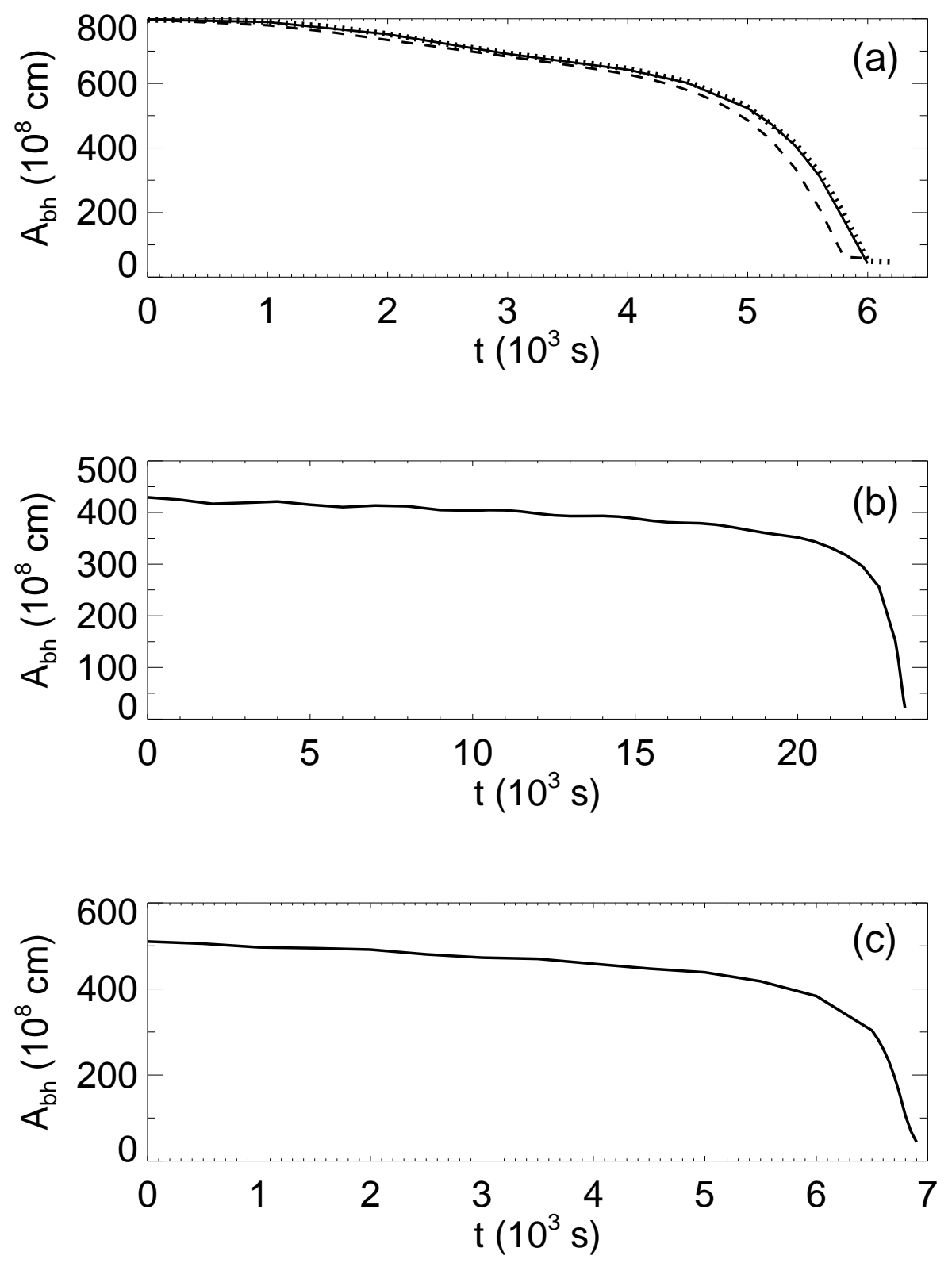

Fig. 7.- The distance between the compact remnant and the center of mass is plotted as function of time for different models: (a) model 8A (solid line), 8B (dotted line), and 8C (dashed line); (b) model 4C; (c) model 16A. The compact remnant inspirals from half its initial separation to the center of the helium star in less than $\sim 500 \mathrm{~s}$. 


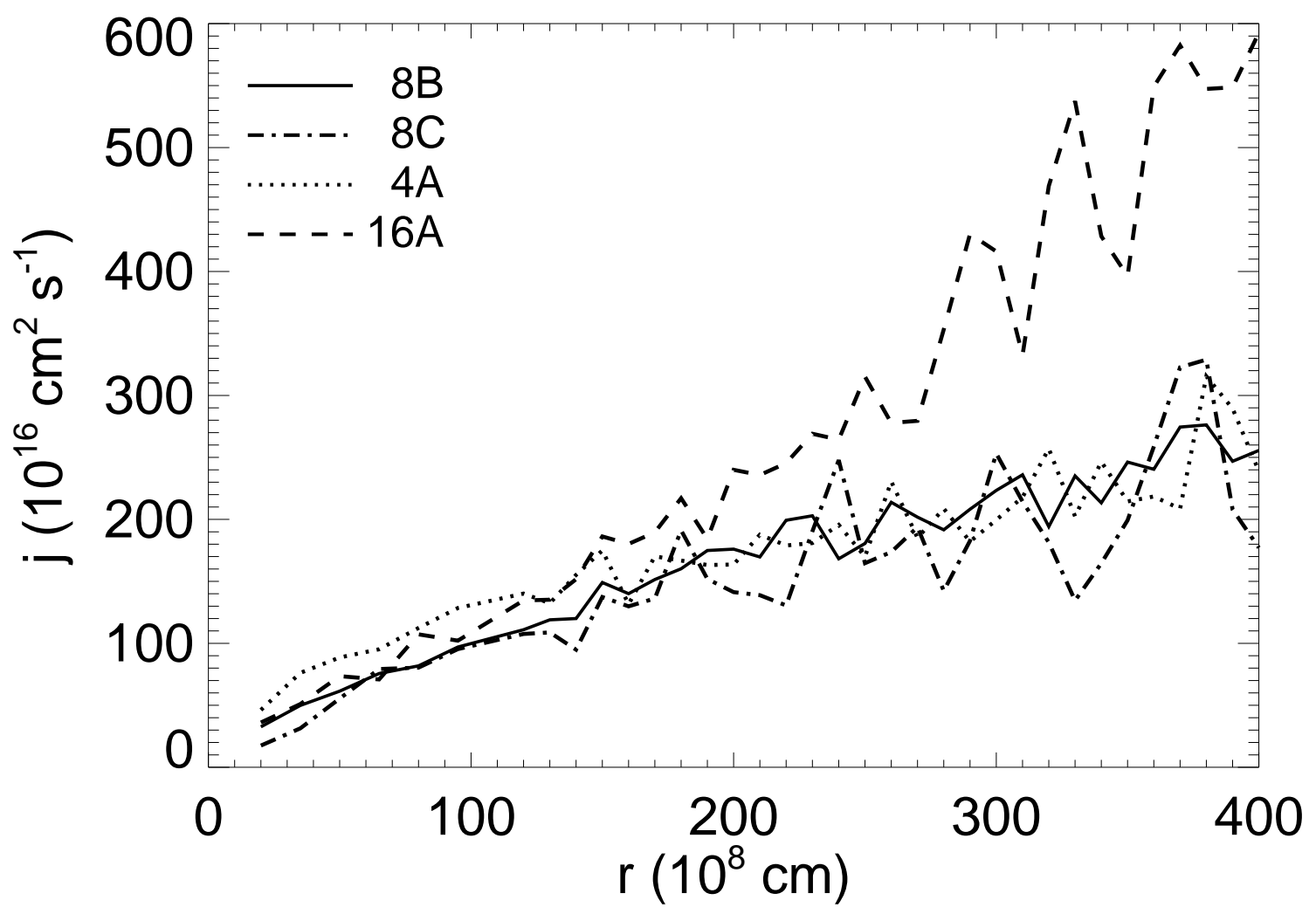

Fig. 8.- Spin angular momentum of the helium core at the time when the compact remnant reaches the center of the core for model $8 \mathrm{~B}$ (solid line), $8 \mathrm{C}$ (dash dot line), $4 \mathrm{~A}$ (dotted line) and 16A (dashed line). 


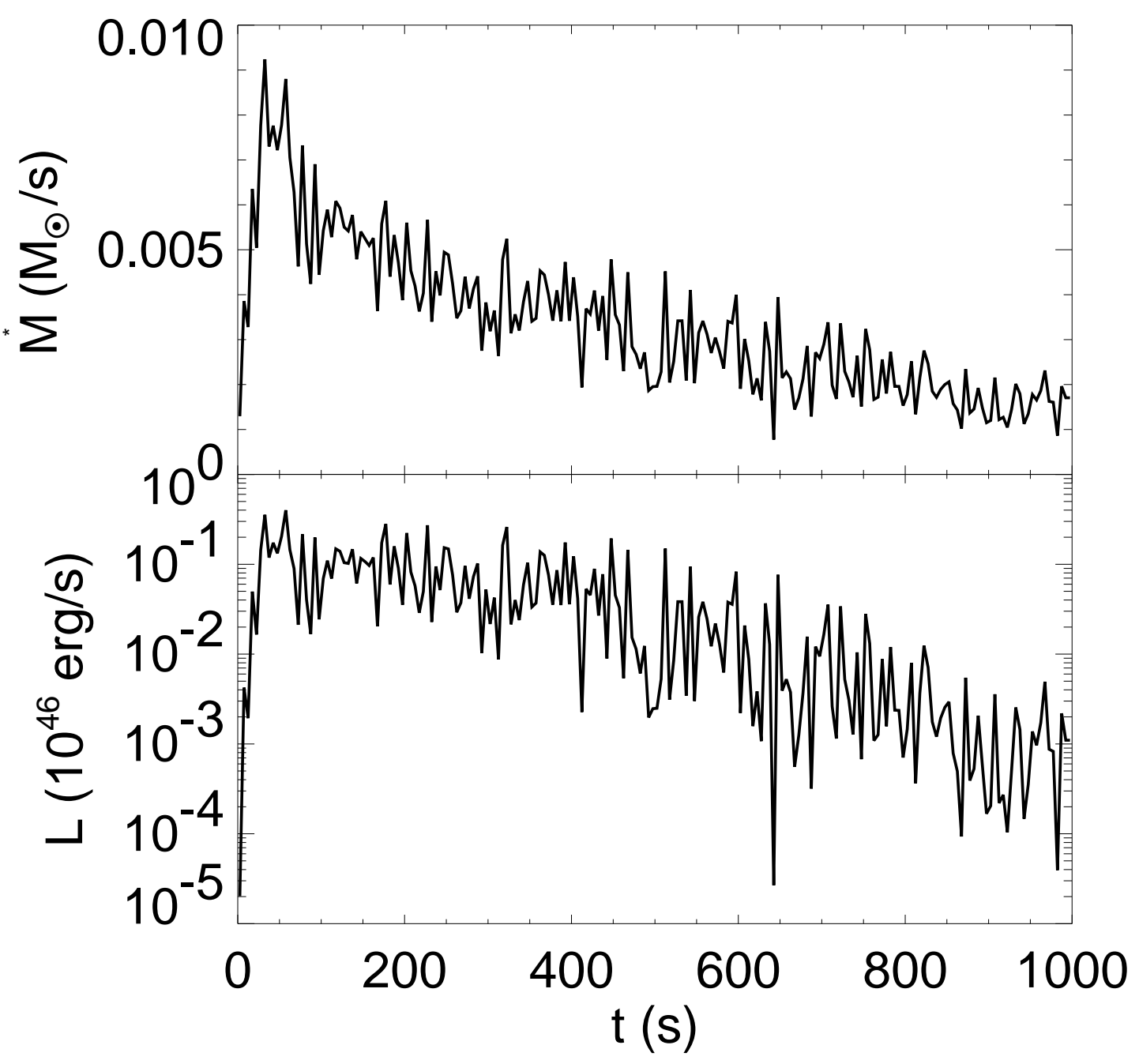

Fig. 9.- Estimates of accretion rate and energy generation rate from neutrino annihilation for model 8B. 


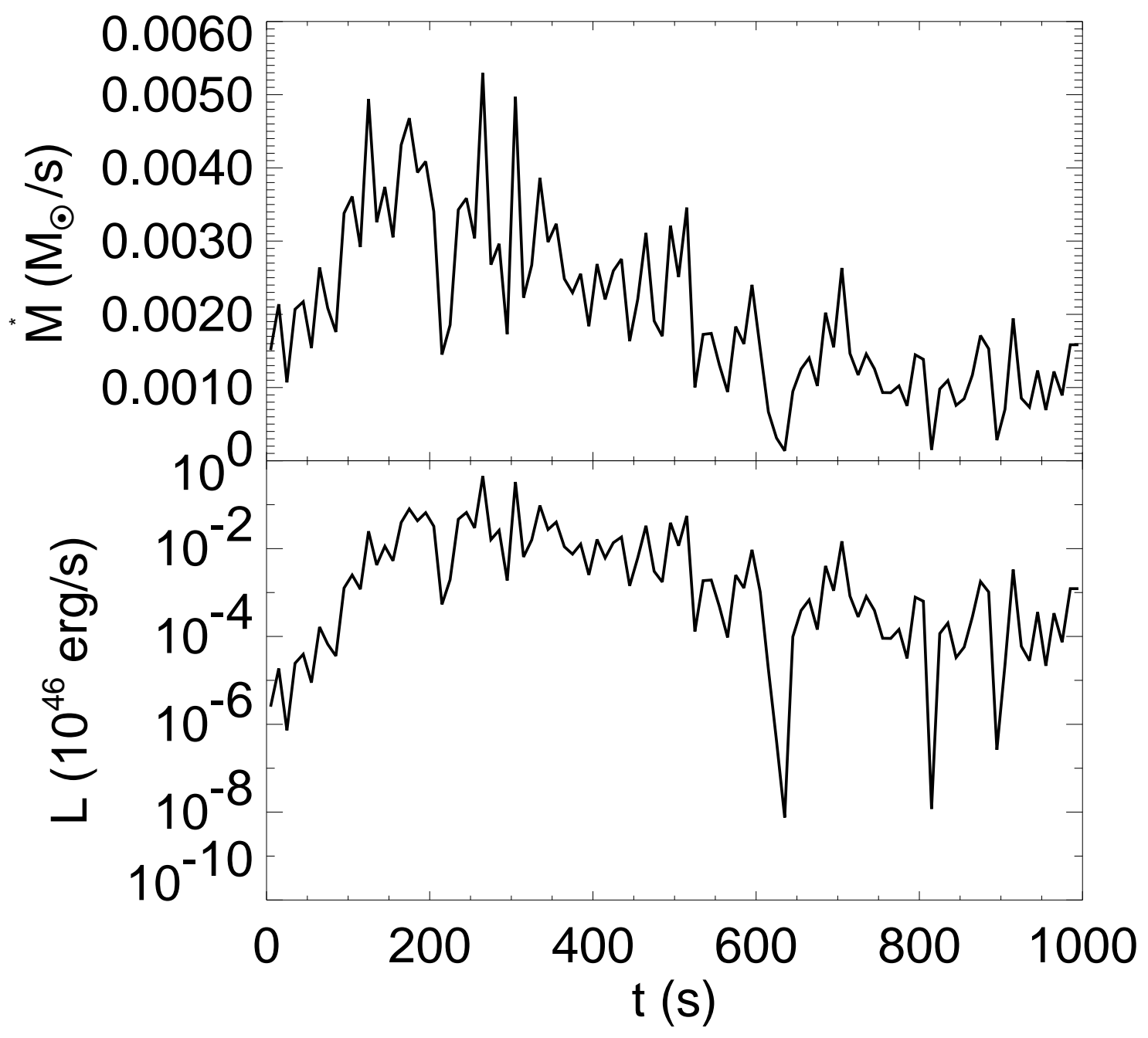

Fig. 10. - Estimates of accretion rate and energy generation rate from neutrino annihilation for model $4 \mathrm{~A}$. 


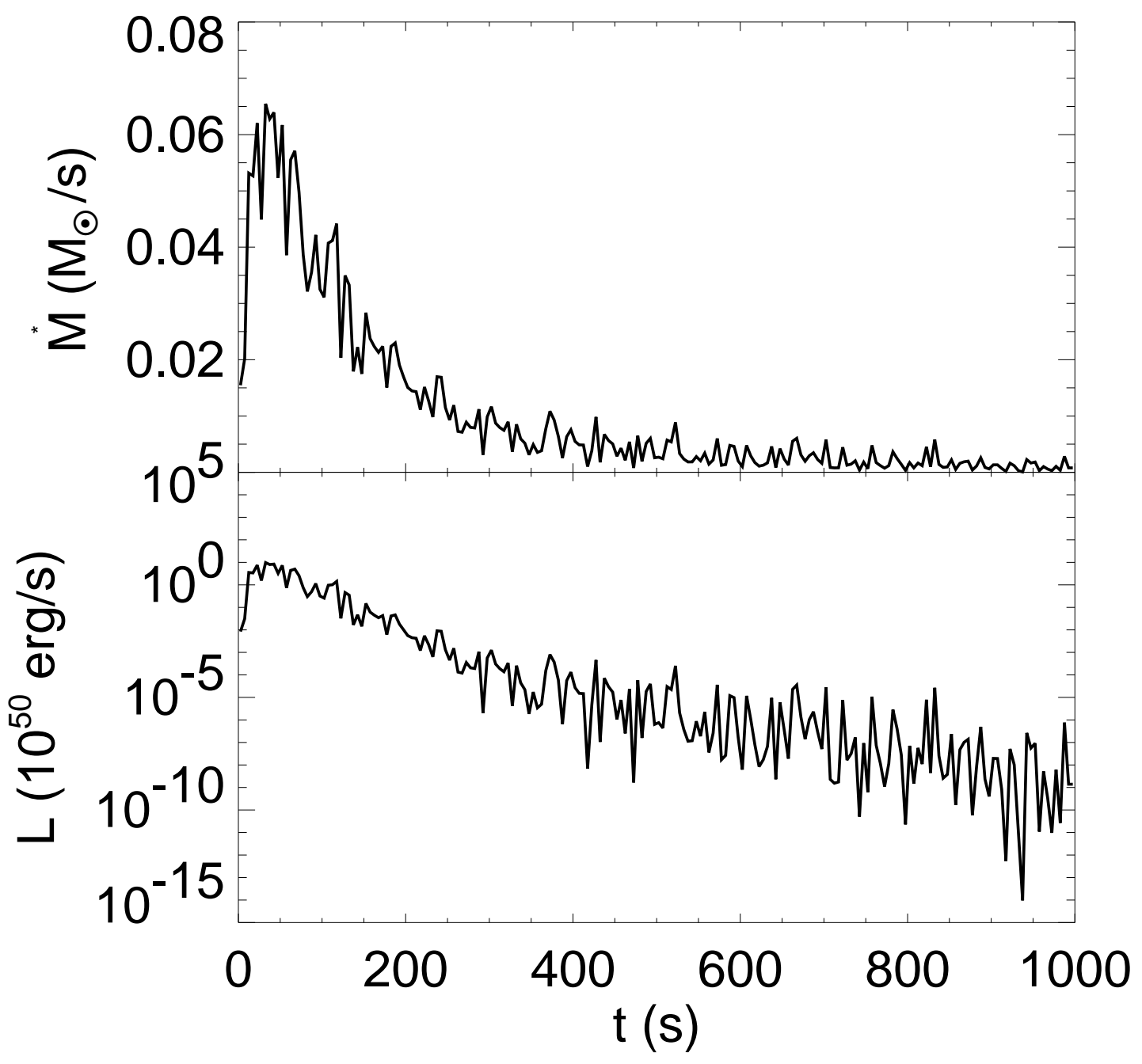

Fig. 11. - Estimates of accretion rate and energy generation rate from neutrino annihilation for model 16A. 


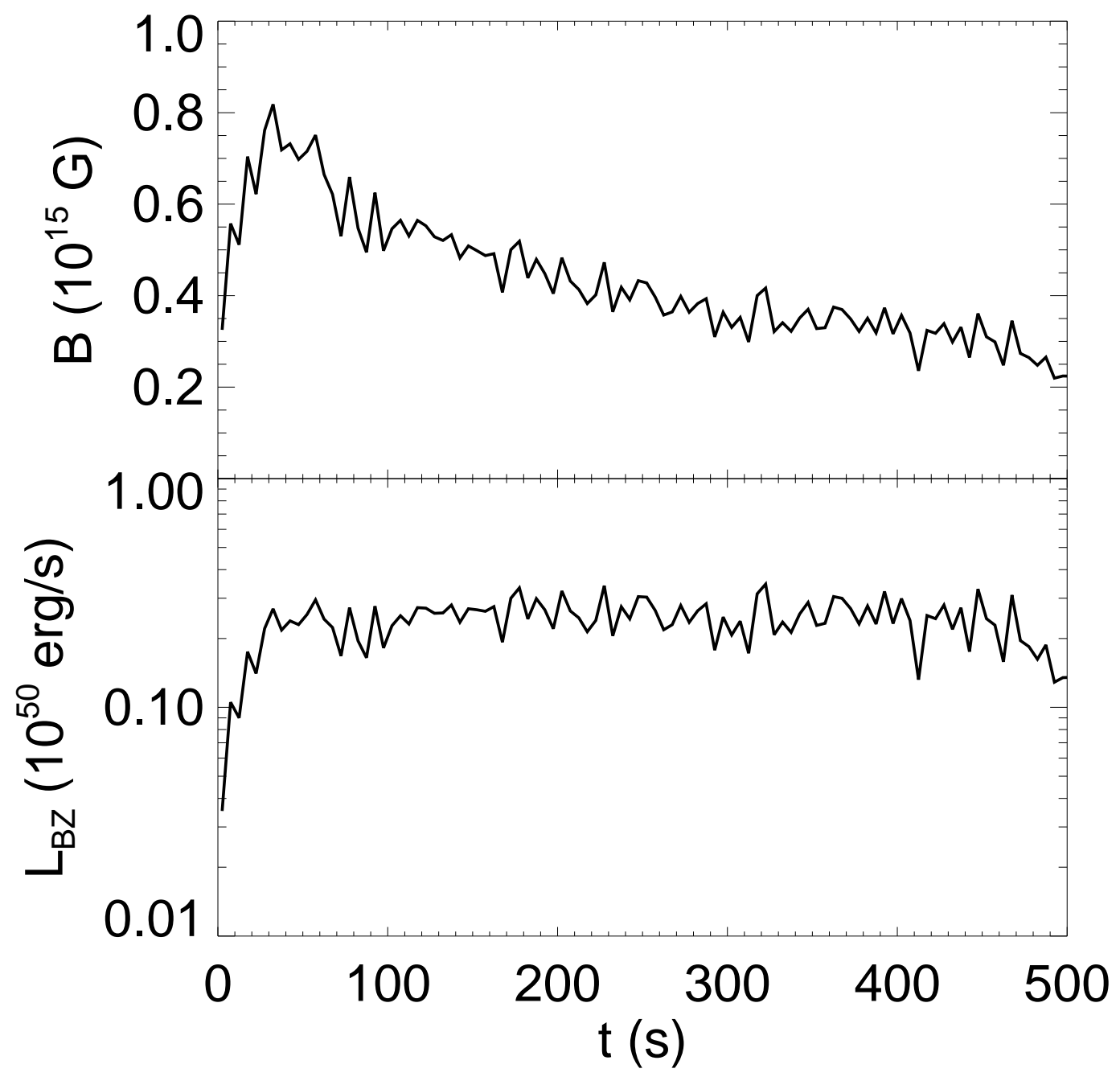

Fig. 12. - Energy deposition rate vs. time from a GRB engine driven by magnetic fields for model 8B. The energy does not drop off dramatically, however, as soon as a burst is launched, it will disrupt the accretion and shut the engine off. These deposition rates assume the magnetic energy density equals the kinetic energy density of the accreting material and is likely to be an overestimate. 